\title{
Baseline Wandering Removal in Optical Mapping Measurements With PID Control in Phase Space
}

\author{
Shaun Eisner ${ }^{1}$, Flavio H Fenton ${ }^{1}$, Ilija Uzelac ${ }^{1}$ \\ ${ }^{1}$ Georgia Institute of Technology, Atlanta, USA
}

\begin{abstract}
Optical imaging methods on ex-vivo hearts have had large impact in furthering our understanding of cardiac electrophysiology. One common problem in this method is a baseline wandering of the fluorescence signals over time, caused by dye photo-bleaching, small variation of the excitation light source, or other similar artifacts. Due to its relative magnitude, the removal of baseline wandering can be a nontrivial task and has major implications for analyzing important physiological dynamics such as traveling waves and alternans. Here we present a computational technique for the removal of such baseline wandering based on Proportional-Integral-Derivative (PID) closed loop feedback. The PID method applied a continuous control stimulus to the input $V_{m}$ based on an error value which is defined by Euclidean distance from a pre-computed setpoint in phase space. We quantify and validate the PID control method by adding a linear combination of arbitrary sinusoidal drift, of frequency less than the signal pacing frequency, to the system signal $V_{m}$. The PID control loop effectively removed the baseline wandering with minimal degradation to the input $V_{m}$, and thus provides a viable tool for baseline wandering removal when implemented in an appropriate phase space. The computational simplicity of the method also lends itself to implementation in embedded systems, such as Arduinos and Raspberry-Pis.
\end{abstract}

\section{Introduction}

Optical imaging with fluorescent dyes is a powerful research tool to study the complex dynamics of cardiac electrical activity[1-3]. O, and can be performed from a single cell[4] to whole heart. $V_{m}$ is one of the most used fluorescent dye. When a $\mathrm{V}_{\mathrm{m}}$ dye is injected circulating through an ex-vivo heart, it binds to the cell membrane. As the fluorescence level of $\mathrm{V}_{\mathrm{m}}$ dyes depends on the cell membrane potential, this important physiological parameter can be imaged with optical mapping methods using high speed cameras, suitable excitation light source, and proper excitation and emission filters[5]. Instability of light sources in terms of light intensity and shifts of excitation wavelength, dye photo-bleaching, dye internalization, and residual cardiac contraction, add to variability of fluorescence baseline. As the baseline drift is in the low frequency range, it is possible to filter out the majority of the drift. Digital filters are commonly used. However, based on the amount of data necessary to process, their implementation on single or even multi core CPUs can only be done for off-line data processing only with long processing times. The aim of this study is to explore the PID closed feedback loop control methods for use in the removal of aforementioned baseline wandering in optical mapping measurements. The simplicity of the methods is suitable for implementation in low power devices, such as embedded systems based on microcontrollers and for systems-on-chip such as Raspberry Pi for real-time processing.

\subsection{PID Control}

PID feedback control is a ubiquitous tool utilized in the control of plant processes which are inherently unstable or prone to significant outside disturbances. Traditionally, the control signal is applied by computing a constant setpoint value $S$, which the system state is continuously compared to at each point in time. An error function $e(t)$ is then computed by evaluating the difference between the system state and setpoint value at each given time step. The control stimulus which is to be applied to the system state is then determined by evaluating a linear combination of the $D^{1}, D^{-1}$, and $D^{0}$ operators acting on the error function $e(t)$.

$$
C(t)=K_{p} e(t)+K_{I} \int_{0}^{t} e\left(t^{\prime}\right) d t^{\prime}+K_{D} \frac{d e(t)}{d t}
$$

Equation (1) is known as the PID equation and expresses the control stimulus as defined above as a function of the error function $e(t)$ and 3 unknown parameters $K_{p}, K_{I}, K_{D}$. 


\section{Methods}

Baseline wandering removal was performed by applying a control stimulus determined according to a PID closed loop feedback control algorithm applied to a specified transform of the system signal, denoted as the baseline function, $B(t)$. Simulated signal data was obtained from a numerical model of an auto-oscillatory FitzHugh-Nagumo system, while experimental data was obtained from optical mapping measurements performed on isolated rabbit hearts stained with Di-4-ANBDQPQ Vm.

\subsection{The Baseline Function}

The baseline function $B(t)$ utilized for the computation of the control stimulus was determined by applying a moving average function to the system signal $V(t)$ over an integer multiple of the period of the system oscillations.

$$
B(t)=\frac{1}{N T} \int_{t-N T}^{t} V\left(t^{\prime}\right) d t^{\prime}
$$

This choice of baseline function allows for a control system which is sensitive only to perturbations of frequency less than the chosen averaging window $N T$, allowing the system to ignore randomized high frequency perturbations or inherent oscillatory behavior in the system dynamics.

In the case of our control system, $N$ was set to a constant value of 2 in order to allow for sensitivity to cardiac alternans, a quasi-oscillatory phenomena with a period twice that of the system oscillatory period, $T$.

Finally, the setpoint value $S$ utilized in computation of the error function was defined as the average of the system signal over the first 2 periods.

$$
S=\frac{1}{2 T} \int_{0}^{2 T} V\left(t^{\prime}\right) d t^{\prime}
$$

\subsection{Control Stimulus}

The PID control stimulus applied to the system signal was determined via the traditional PID algorithm (1) utilizing an error function $e(t)$ defined as the difference between the baseline function $B(t)$ and the setpoint value $S$.

$$
e(t)=B(t)-S
$$

Determination of the PID coefficients was done in accordance with John Shaw's [6] description of the classic Ziegler-Nichols Method [7], for the tuning of the PID coefficients in feedback control systems. The Ziegler-Nichols method is performed first by setting each of the $K_{P}, K_{I}$, and $K_{D}$ coefficients to 0 . Next, the $K_{P}$ value is increased until the application of a $5 \%$ change to the setpoint value results in sustained oscillations in the system signal. The
Table 1. Ziegler-Nichols PID coefficient values

\begin{tabular}{lcc}
\hline \hline Coefficient & Ziegler-Nichols Value & Computed Value \\
\hline$K_{P}$ & $0.6 G_{u}$ & -8.00 \\
$K_{I}$ & $2 / t_{u}$ & -0.0033 \\
$K_{D}$ & $t_{u} / 8$ & -75.00 \\
\hline \hline
\end{tabular}

resulting value of $K_{P}$ at which oscillations occur is the ultimate gain $G_{u}$ and the period of oscillations is the ultimate period $t_{u}$. This results in the values listed in Table 1 .

\section{Results}

The accuracy of the PID control method was determined using a combined quantitative and qualitative approach. First, a system signal corrected with the PID algorithm was compared to an ideal undrifted signal by computing the percent change in the baseline over the entire time interval during which system signal was measured. This resulted in a $0.0488 \%$ error as compared to the ideal signal. In addition, a correlation function was computed as the bivariate correlation coefficient $p$ for a time interval of $[t-2 T, t]$ where $T$ is the period of system oscillations, for all times $t$ during which the system signal was measured. This was done in order characterize changes in morphology as compared to the ideal signal. Figure 5 depicts this correlation function over time. It should be noted that as $t \rightarrow \infty$, the correlation value begins to asymptotically approach a value close to 0.99 indicating a resulting $1 \%$ steady state change to signal morphology. Next, the same correlation function and percent change in baseline were computed for a signal with an arbitrary combination of linear and sinusoidal drift. This result is depicted in Figure 5. Using this technique, the drifted signal underwent a $2.41 \%$ change in baseline, indicating a $2.41 \%$ difference as compared to the ideal signal while the corrected signal only underwent a $0.00255 \%$ change, indicating a $0.00255 \%$ error as compared to the undrifted signal. In addition, the corrected signal maintained a $1 \%$ steady state error in morphology as compared to the undrifted signal, indicating that any resulting steady state error in morphology is not a function

of the drift, but possibly of the PID parameter values and choice of period used in the computation of the baseline function.

In addition to the correlation function and baseline error values, accuracy of PID corrections were qualitatively determined via analysis of the frequency spectrum of the pre-corrected and post-corrected signals. As indicated in figure 6 , the simulated data set experienced a reduction in frequency components less than $2 \mathrm{~Hz}$ as compared to the uncorrected data set. As baseline drifting is a phenomena which most often occurs at frequencies lower than times 


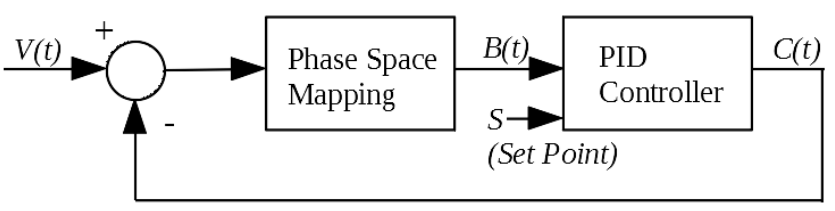

Figure 1. Block diagram of the algorithm for baseline removal as a control system. Summing block serves as an actuator driven with control signal input, and the signal difference if mapped into phase space by corresponding baseline function. The error function is defined as a difference between $\mathrm{B}(\mathrm{t})$ state and the setpoint $\mathrm{S}$, upon which $\mathrm{PID}$ controller calculates the control signal $\mathrm{C}(\mathrm{t})$
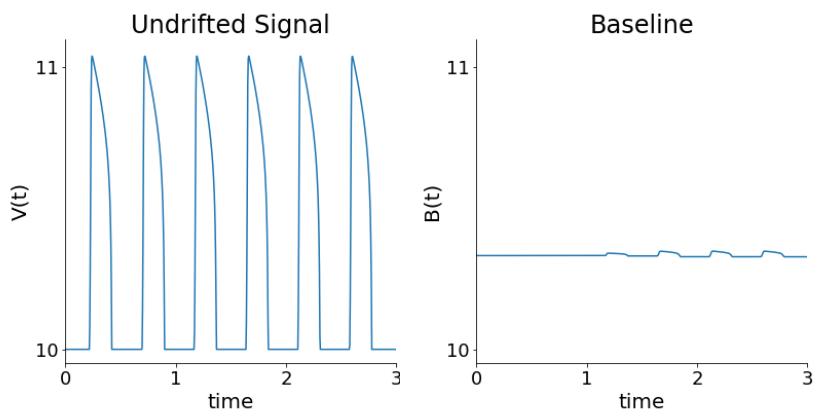

Figure 2. Depicts the time series of a simulated system voltage signal (Left) and its corresponding baseline function (Right)
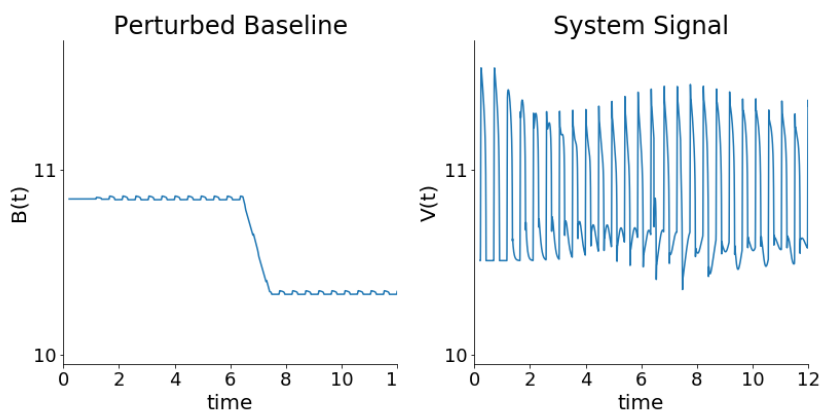

Figure 3. Depicts the plot of a setpoint value perturbed by $5 \%$ at time $t=6 s$ (Left) and the resulting oscillations in the Controlled system signal for $K_{P}=G_{u}$ (Right)
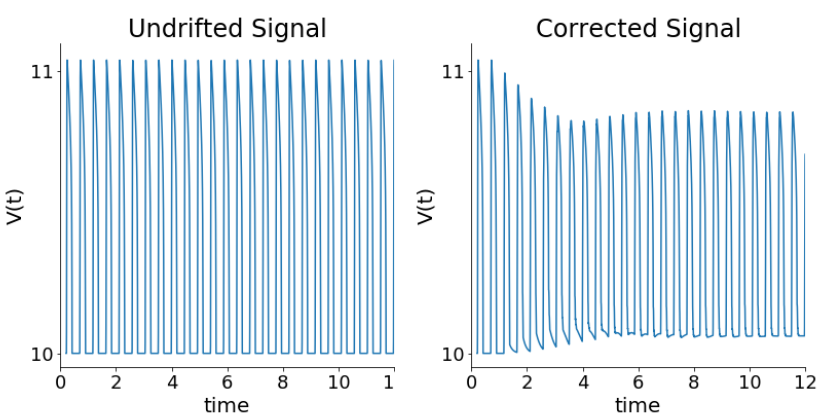

Figure 4. Depicts the time series of the undrifted (Left) and corrected (Right) system signal corresponding to the correlation function in Figure 5
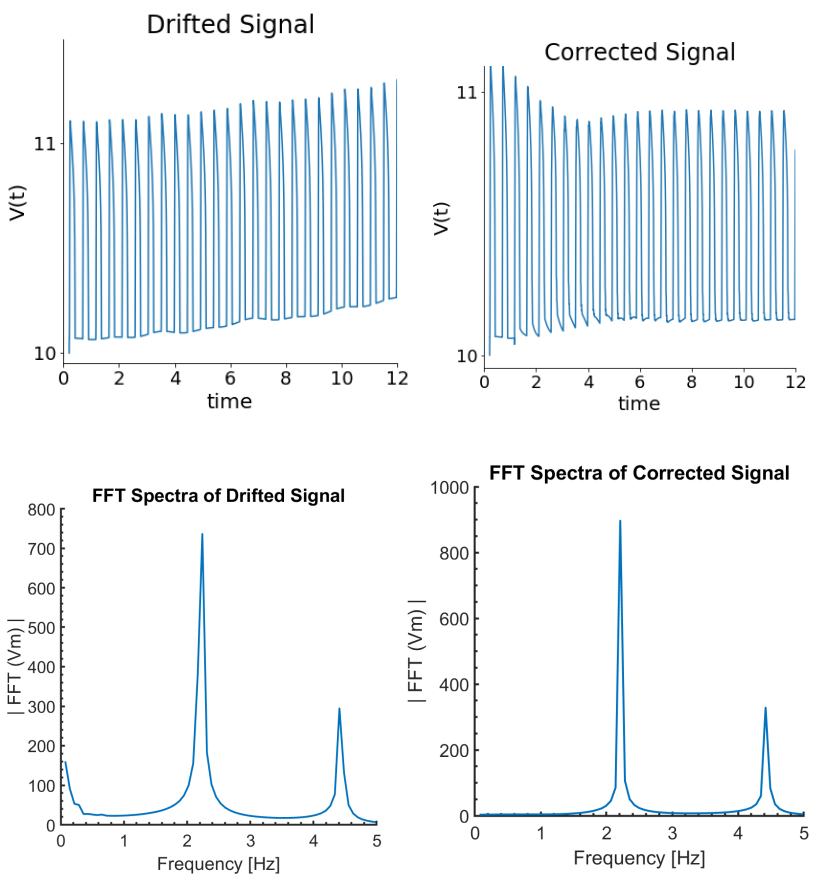

Figure 5. Depicts the baseline function $B(t)$ of a drifted (Left) and corrected (Right) system signal over time, with corresponding FFT frequency spectra
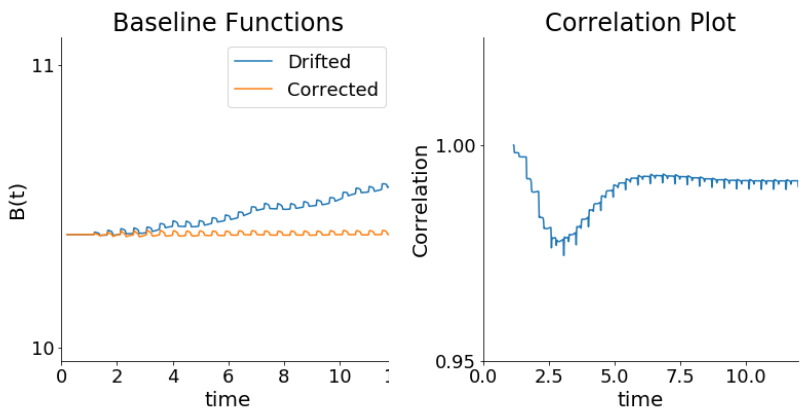

Figure 6. Depicts the baseline function $B(t)$ of a drifted and corrected system signal (Left) as well as the plot of a correlation function for a correction applied to an undrifted system signal (Right) 
the frequency of oscillatory dynamics in the chosen system, this loss of low frequency components further demonstrates a reduction in the baseline drift of the chosen data set.

\section{Discussion}

Baseline drift removal is a first step in the processing of optical mapping data. For short time recordings, on the scale of seconds, baseline drift can be considered linear. In contrast, for long term recordings the baseline drift tends to be highly non-linear. The presented fluorescence baseline filtering methods is robust. In the PID controller optimization, the controller parameters can be obtained using widely accepted Ziegler-Nichols' closed loop method. Overall, the utilization of PID feedback control when applied to an appropriate choice of baseline function proves to be a viable method for the detection and removal of baseline wandering in optical mapping measurements.

\section{Conclusions}

In this study, we presented a method for fluorescence baseline removal. The presented filtering method is robust and can be used in general for optical mapping data as the first step in data pre-processing pipeline prior to subsequent features extraction. In the PID control loop, the PID control parameters can be obtained by perturbation on the mapped signal in its representative phase space. While the frequency (period) of the input signal is needed for phase space mapping, the frequency is not a dominant parameter for effective PID control loop. Longer integration times in phase space mapping make the PID control loop more sensitive to small baseline changes, but at the expense of longer settling time, which is not an issue for long term recordings. The simplicity of the PID control allows its implementation for the embedded systems and lower power devices especially for studies requiring real-time data pro- cessing.

\section{Acknowledgments}

This study was supported with NSF 1762553, and NIH 1R01HL143450-01 grants.

\section{References}

[1] Efimov IR, Nikolski VP, Salama G. Optical imaging of the heart. Circulation research 2004;95(1):21-33.

[2] Pertsov AM, Davidenko JM, Salomonsz R, Baxter WT, Jalife J. Spiral waves of excitation underlie reentrant activity in isolated cardiac muscle. Circulation research 1993;72(3):631650.

[3] Cherry EM, Fenton FH. Visualization of spiral and scroll waves in simulated and experimental cardiac tissue. New Journal of Physics 2008;10(12):125016.

[4] Entcheva Emilia (Washington DUKAAVU. Automated system for high-throughput all-optical dynamic electrophysiology May 2019;(Patent 20190137398).

[5] Uzelac I, Ji YC, Hornung D, Schröder-Scheteling J, Luther S, Gray RA, Cherry EM, Fenton FH. Simultaneous quantification of spatially discordant alternans in voltage and intracellular calcium in langendorff-perfused rabbit hearts and inconsistencies with models of cardiac action potentials and ca transients. Frontiers in physiology 2017;8:819.

[6] John A. The pid control algorithm: How it works how to tune it and how to use it 2nd edition. Process Control Solutions 2003;

[7] Ziegler JG, Nichols NB. Optimum settings for automatic controllers. trans ASME 1942;64(11).

Address for correspondence:

Shaun Eisner

seisner6@gatech.edu

837 State St, School of Physics, Atlanta, GA 30332, USA 